\title{
LA FORMA Y LA ESENCIA: EFECTOS DE LAS LEYES DE TRANSPARENCIA, ACCESO A LA INFORMACIÓN Y PARTICIPACIÓN CIUDADANA EN LA GOBERNANZA DEMOCRÁTICA LOCAL EN CHILE*
}

\author{
José Hernández Bonivento \\ Universidad Autónoma de Chile \\ jhernandez@ichem.cl
}

\section{RESUMEN}

Chile ha introducido reformas legales enfocadas hacia la transparencia, el acceso a la información (Ley 20.285) y la participación ciudadana en la administración pública (Ley 20500). Las evaluaciones de la implementación de estas leyes solamente han medido el cumplimiento de las exigencias legales, sin observar sus efectos en la gobernanza democrática a nivel municipal. Este analiza los efectos de los mecanismos de transparencia, acceso a la información y participación ciudadana en tres ciudades: Puerto Montt, Coyhaique y Punta Arenas. Los resultados revelan que, aún en casos de alto cumplimiento formal de la ley, existen grandes desafíos, tanto de forma como de fondo, que evitan y, en ocasiones, desincentivan el surgimiento de una gobernanza democrática a nivel local. Al mismo tiempo, este artículo muestra que la implementación de estas leyes aunque también han generado espacios para el surgimiento de iniciativas locales que han dinamizado la interacción entre las municipalidades y la sociedad civil a nivel local.

Palabras clave: Transparencia, Participación, Gobiernos locales, Chile.

* Este artículo ha sido elaborado con apoyo del Proyecto Interno DIP 44-2015 de la Universidad Autónoma de Chile y del Proyecto Fondecyt Iniciación No. 11170216 de CONICYT, Gobierno de Chile. 


\title{
FORM AND ESSENCE: THE EFFECTS OF LAWS ON TRANSPARENCY, ACCESS TO INFORMATION AND CITIZEN PARTICIPATION IN LOCAL DEMOCRATIC GOVERNANCE IN CHILE
}

\begin{abstract}
In recent years, Chile has implemented legal reforms on transparency, access to information (Law 20.285) and citizen participation in public administration (Law 20.500). Evaluations of the implementation of these laws have only measured compliance with legal requirements, without examining their effects on democratic governance at the municipal level. Looking at theoretical and methodological aspects, this article analyzes the effects of institutional mechanisms for transparency, access to information and citizen participation in three regional capitals of Chilean Patagonia: Puerto Montt, Coyhaique and Punta Arenas. The results reveal that, even in cases of high formal compliance, there are great challenges, both in form and substance, which hamper the emergence of local democratic governance. At the same time, the article also finds evidence of the emergence of local collaborative initiatives, which have improved interaction between municipal governments and civil society at the local level.
\end{abstract}

Keywords: Transparency, Participation, Local governments, Chile. 


\section{INTRODUCCIÓN}

Durante la última década, América Latina se han embarcado en varios procesos institucionales que buscan fomentar un gobierno más transparente y abierto hacia la ciudadanía. De la mano de esfuerzos internacionales, como lo ha sido la Alianza para el Gobierno Abierto (OGP por sus siglas en inglés), organización internacional a la que pertenecen 17 países de la región y que promueve los principios de participación ciudadana, transparencia de la administración pública y rendición de cuentas, no han sido pocos los países que han realizado esfuerzos por crear o modernizar sus aparatos institucionales en busca de dichos objetivos. Y Chile no ha sido la excepción.

En efecto, desde antes de integrarse al conjunto de países de la OGP, Chile ya había aprobado leyes que buscaron abrir y acercar la administración pública, en especial la municipal, a la ciudadanía y a su entorno. El surgimiento de las leyes de transparencia y acceso a la información (20.285) y de asociaciones y participación ciudadana en la gestión pública (20.500) han buscado establecer nuevos parámetros formales de interacción entre las administraciones locales y sus comunidades.

Pero ¿es el cumplimiento formal de dichas leyes suficiente para alcanzar metas de gobernanza democrática local? ¿Cuáles han sido los efectos de estos marcos institucionales en la forma como la administración local se relaciona con su comunidad? Con estas preguntas en mente, el objetivo central del presente texto es analizar si la implementación de los distintos mecanismos de transparencia, acceso a la información y participación ciudadana, establecidos por la legislación nacional chilena en la última década, han generado cambios significativos en la interacción entre las administraciones municipales y la ciudadanía, y si dichos cambios han implicado mejoras en la gestión local y en la consolidación de valores democráticos a escala local.

Para ello, se expone en un primer apartado cómo, según las teorías de gobernanza local, un gobierno más abierto, transparente y participativo debería mejorar las acciones estatales y apoyaría la consolidación de valores democráticos a escala municipal. En la segunda parte exponemos el diseño metodológico de la presente investigación, basado en análisis cualitativos de tres ciudades de la Patagonia chilena: Coyhaique, Puerto Montt y Punta Arenas. En la tercera parte se presentan los resultados más evidentes del trabajo en terreno, para terminar con un apartado de discusión sobre las implicancias del caso chileno a la teoría y el estudio de la gobernanza democrática local. 


\section{MARCO TEÓRICO: LA GOBERNANZA DEMOCRÁTICA LOCAL}

El término gobernanza es utilizado de manera generalizada en el ámbito de la administración pública como un nuevo modelo de gobernación y dirección social, el cual va más allá de la administración burocrática y la llamada Nueva Gestión Pública, para centrarse en mecanismos de interacción entre actores sociales (llámense entidades estatales, organizaciones sociales, empresas privadas o ciudadanía no organizada), todo esto con la finalidad de definir objetivos y alcanzar metas de beneficio colectivo a través de la colaboración y la co-creación de acciones públicas (Prats 2005, Aguilar 2008, Osborne 2010, Levi-Faur 2012).

Dicho modelo de gestión pública buscaría, en un nivel ideal-normativo, la construcción de redes entre los diversos actores sociales con el interés de consolidar una regulación pública, entendida como la generación de valores, estándares, planes, reglas y decisiones concretas que tengan como objetivo principal mejorar la situación del entorno de la red en su conjunto (Torfing 2012). Implica esto la necesidad de establecer mecanismos de interacción entre los gobiernos y las administraciones públicas con los actores sociales que, de manera inevitable en nuestros tiempos de complejidad, han cobrado una importancia social, económica y política demasiado alta como para obviarlos del proceso de dirección social (Kooiman 2003, Peters 2010).

Esta idea de interacción constante se vuelve central cuando hablamos del nivel local de gobierno: en efecto, aunque muchos autores han trabajado el concepto de gobernanza local de manera diversa, sea con acercamientos conceptuales (Navarro Gómez 2002, Navarro 2004, Carmona 2005), o en situaciones específicas (Navarro y Ramírez 2005, Paredes 2007, Yilmaz, Beris, y Serrano-Berthet 2008, Varela Álvarez 2010, UNPD 2010). En todos estos casos se remarca la importancia de la participación ciudadana y la deliberación entre actores sociales para el buen funcionamiento de los gobiernos locales, sobre todo en términos de gobernanza democrática (Young 2002, Fung 2006, 2015), dando prioridad al diálogo, el debate y la deliberación entre actores sociales para alcanzar una doble meta: mejorar las acciones estatales a través de la retroalimentación ciudadana y el aprovechamiento de capacidades de otros sectores, al mismo tiempo que consolidar los principios democráticos de participación y deliberación ciudadana frente problemas comunes.

Al revisar la literatura, es posible detectar tres premisas esenciales para la gobernanza democrática de las administraciones públicas locales (Tabla 1). La primera de ellas es la implementación de una gestión de proximidad, 
donde las administraciones locales se encarguen de recoger de primera mano las demandas, los intereses y las necesidades de la ciudadanía, lográndose de esta forma la priorización de las inquietudes ciudadanas y la construcción colaborativa de acciones que permitan alcanzar metas de común beneficio (Blanco y Gomá 2002, Font 2007, Subirats 2007, Brugué y Gallego 2007, Villoria 2010). Esta idea de la proximidad sigue siendo pie fundamental para los discursos a favor de la descentralización política, fiscal y administrativa, pues conllevaría el fortalecimiento democrático, a partir de una mayor representación de los intereses locales, así como mejor asignación de recursos partiendo del involucramiento ciudadano en la esfera local. Ahora, el gran desafío que enfrenta dicha premisa, sobre todo en el contexto latinoamericano, es la construcción de capacidades administrativas que permitan potenciar las posibilidades de la descentralización del Estado (Mascareño 2008, Salvador Crespo 2008, Falleti 2010), así como la necesidad de generar incentivos al interior de las administraciones locales para que aprovechen la interacción constante entre sus propios funcionarios, la ciudadanía y las distintas administraciones públicas que intervienen en sus territorios (Goss 2001).

La segunda premisa es la gestión transparente de la administración pública: muy de la mano del concepto de gobierno abierto, la gestión transparente busca entregar a la ciudadanía toda la información sobre los asuntos públicos sin bloqueos innecesarios, buscando la consolidación de un ciudadano más empoderado, conocedor de los asuntos públicos y capaz de hacer seguimiento a las acciones estatales para exigir cuentas a las entidades de gobierno. Esta idea del ciudadano empoderado implicaría un mejoramiento de las acciones estatales al desincentivar prácticas patrimonialistas y corruptas en entidades estatales (Lathrop y Ruma 2010, Meijer, Curtin y Hillebrandt 2012, Yarvuz y Welch 2014, Lourenco 2015). Al mismo tiempo, una ciudadanía informada y empoderada permitiría generar círculos virtuosos para la deliberación pública (Worthy 2010), donde la ciudadanía cuenta con mayores herramientas para debatir y construir su propio poder comunicativo (por usar el término de Habermas) que permita hacer contrapesos al poder administrativo. Por último, la idea de un gobierno local transparente también permitiría la consolidación de comunidades interpretativas a nivel local (Conford et al. 2013), las cuales se convierten en interlocutores constantes que no solo manejan datos públicos, sino que los construyen e interpretan, generando una mayor y mejor deliberación pública.

Por último, está la búsqueda de una gestión participativa: con ella se busca entonces establecer diálogos constantes y colaborativos entre gobiernos y ciudadanos empoderados para mejorar tanto la legitimidad de 
las acciones de la administración como la efectividad misma del quehacer público (Harrison y Sayogo 2010, Evans y Campos 2013). Para ello, se establecen arreglos institucionales y mecanismos de interacción entre la administración y la sociedad civil, entendiendo que el mismo acto de participar en estos espacios logra generar conciencia sobre la importancia de la democracia y del involucramiento ciudadano, así como despertar interés en las cuestiones públicas de su propia comunidad (Michels y De Graaf 2010, Geurtz y van de Wijdeven 2010). Esto explica el surgimiento de una gran variedad de leyes, mecanismos y estructuras formales que buscaron el involucramiento ciudadano y la consolidación de valores democráticos en el nivel local a través de la participación ciudadana (McNulty 2013), una tendencia que se materializó tanto en reformas constitucionales como en leyes, programas y prácticas locales, como fueron los presupuestos participativos de Porto Alegre, un caso que algunos llegaron a considerar como el surgimiento de una nueva democracia participativa y redistributiva (De Sousa Santos 2005).

Tabla 1: Potenciales resultados de la gobernanza democrática local

\begin{tabular}{ccc} 
Premisa teórica & Fortalecimiento democracia & Mejoramiento acción estatal \\
\hline Proximidad & $\begin{array}{c}\text { Mayor representación de } \\
\text { intereses locales }\end{array}$ & $\begin{array}{c}\text { Priorización acciones y } \\
\text { mejoramiento asignación de } \\
\text { recursos }\end{array}$ \\
Transparencia & $\begin{array}{c}\text { Rendición de cuentas } \\
\text { constante y empoderamiento }\end{array}$ & $\begin{array}{c}\text { Mejora la retroalimentación y } \\
\text { evidencia acciones de corrupción } \\
\text { Participación }\end{array}$ \\
& $\begin{array}{c}\text { Consolidación de } \\
\text { mecanismos de voz e } \\
\text { involucramiento ciudadano }\end{array}$ & $\begin{array}{c}\text { Posibilidades de colaboración } \\
\text { entre sectores sociales }\end{array}$
\end{tabular}

Fuente: Elaboración propia.

\section{GOBERNANZA LOCAL Y EL CASO CHILENO}

La presente investigación parte de estas premisas para analizar el caso chileno, el cual, por varias razones, se presenta como relevante para observar los efectos de la legislación nacional en la gobernanza local.

En primer lugar, es considerado el país con mayor nivel de desarrollo de sus administraciones públicas: según el diagnóstico realizado por el Banco Interamericano de Desarrollo, el servicio civil chileno es el que obtiene los mejores resultados, por encima de grandes burocracias como la brasileńa y la mexicana, y se considera un ejemplo a seguir para la región (Iacoviello y Strazza 2014). Al mismo tiempo, Chile es también visto como un ejemplo 
de política programática (donde la asignación de bienes públicos se realiza a través de políticas públicas y no de redes clientelares partidistas) frente a otros países latinoamericanos, donde las relaciones clientelares pueden llegar a remplazar la misma institucionalidad nacional (Calvo y Murillo 2013). Se entiende entonces que existe un alto desarrollo institucional en el país, lo que haría pensar que los cambios institucionales deberían generar cambios visibles en la realidad. Por último, el carácter centralista de las políticas públicas chilenas, donde las acciones estatales se diseñan de forma sectorial (a nivel ministerial) desde Santiago y de manera uniforme para todos los territorios del país (Olavarría 2010, Vial 2016), permite comparar la implementación de las políticas de transparencia y participación ciudadana entre diversos territorios, buscando detectar aspectos no formales que influyan en la implementación de dichas políticas.

Ahora, lo más relevante para el presente estudio del caso chileno es el surgimiento de dos leyes nacionales que buscan fomentar el involucramiento ciudadano en la gestión pública: la Ley 20.285 sobre acceso a la información pública, expedida en 2008, y la Ley 20.500 sobre asociaciones y participación ciudadana en la gestión pública, que surge en 2011.

La primera de ellas (Ley 20.285) establece el acceso a la información pública como un derecho ciudadano, generando herramientas tanto para la transparencia activa (información que debe ser publicada y actualizada por todas las administraciones del país) como para la transparencia pasiva (parámetros para las solicitudes de información formuladas por la ciudadanía). Además, crea el Consejo para la Transparencia (CPLT), agencia independiente encargada de hacer seguimiento a la implementación de la ley que, además, cuenta con capacidad de sancionar el incumplimiento. En cuanto a su implementación, según los reportes del CPLT para el 2017 se había alcanzado un cumplimiento promedio del $74,21 \%$ en transparencia activa en las municipalidades, más del doble del 30,26\% con el que partió la medición en el 2012, y en cuanto a transparencia pasiva, la última medición arrojó un promedio de $80,51 \%$ de cumplimiento a nivel municipal (Consejo para la Transparencia 2017).

En cuanto a participación ciudadana, la Ley 20.500 establece el derecho a la participación ciudadana tanto en las políticas como en los diversos planes y programas del Estado, estableciendo que cada municipalidad debe contar con una ordenanza de participación ciudadana que regule todos los mecanismos formales de involucramiento ciudadano, creando a su vez la obligatoriedad de establecer en cada municipalidad un Consejo Comunal de la Sociedad Civil (COSOC) como principal espacio consultivo para la 
participación de organizaciones sociales ante la municipalidad. Sobre estos mecanismos se han desarrollado algunos análisis, los cuales coinciden en que, a pesar de algunos avances puntuales, son evidentes las deficiencias en el diseño de estos mecanismos, en especial su carácter consultivo y su baja articulación con la agenda de política pública (Delamaza et al. 2012, Consejo Nacional de Participación Ciudadana 2017, Escoffier 2017). En cuanto al cumplimiento de la ley a nivel municipal, el Sistema Nacional de Información Municipal (SINIM) señala que el 73,13\% de las municipalidades contaba con ordenanza de participación y el 65,5\% de estas contaba con COSOC para el año 2017 (SINIM 2018).

Se observa, entonces, que existe un cumplimiento relativamente alto y generalizado en todo el país de las leyes de participación y transparencia a nivel municipal, lo cual permite realizar una mirada más cercana sobre los efectos de dichas leyes sobre la gobernanza municipal.

\section{METODOLOGÍA DE INVESTIGACIÓN}

Tomando como ejemplo otras investigaciones que evalúan los efectos de los mecanismos participativos en la gobernanza local (Geurtz y van de Wijdeven 2010, Michels y De Graaf 2010), se plantea una investigación de carácter cualitativo con estudio de casos. Dada la diversidad geográfica, social y poblacional de las municipalidades chilenas, y buscando generar comparaciones por similitud en términos sociales y poblacionales, y por diferencia en cuanto al cumplimiento de la legislación en transparencia y participación ciudadana, se establece como categoría de revisión las veintitrés ciudades intermedias de Chile, entendidas como aquellas ciudades que son foco de desarrollo e intermediación entre las políticas nacionales y los diversos territorios (Maturana y Rojas 2015). En este sentido, la importancia de estas ciudades reside en su protagonismo en el contexto territorial en el que se encuentran (capitales regionales, capitales provinciales y focos económicos o turísticos), convirtiéndose en potenciales multiplicadores de buenas prácticas en los territorios. Partiendo del nivel de cumplimiento legal entre dichas municipalidades, se seleccionan tres casos de estudio: Punta Arenas (Región de Magallanes), Coyhaique (Región de Aysén) y Puerto Montt (Región de Los Lagos) (Tabla 2). 
Tabla 2: Mecanismos de transparencia y participación ciudadana en los casos seleccionados

\begin{tabular}{ccc} 
Municipalidad & $\begin{array}{c}\text { Transparencia } \\
\text { activa 2016 }\end{array}$ & $\begin{array}{c}\text { Mecanismos de participación } \\
\text { en ordenanzas municipales }\end{array}$ \\
\hline Punta Arenas & $89 \%$ & 6 mecanismos \\
Puerto Montt & $0 \%$ & 19 mecanismos \\
Coyhaique & $94 \%$ & 13 mecanismos
\end{tabular}

Fuente: Elaboración propia a partir de Ranking Transparencia Activa CPLT (2016) y las ordenanzas municipales de participación ciudadana (2016).

En cuanto a las diferencias de cumplimiento, el caso de Punta Arenas, se observó un elevado cumplimiento en los temas de transparencia y acceso a la información pública, pero en el momento de revisar su ordenanza municipal de participación ciudadana se observó un bajo desarrollo de mecanismos institucionales de participación ciudadana, contando únicamente con los cuatro mecanismos mencionados en la legislación (Audiencias Públicas, Oficinas de Información y Reclamos, Consejo de la Sociedad Civil y plebiscitos municipales) más dos mecanismos de información (sondeos de opinión e información por páginas web). Por su lado, el caso de Puerto Montt fue el único con un puntaje de 0 puntos en el Ranking de Transparencia Activa Municipal del CPLT en 2016, mientras su ordenanza de participación daba cuenta de hasta 19 mecanismos de participación institucional, el mayor número entre las ciudades de la categoría intermedia, que van desde cabildos abiertos, pasando por consejos comunales en terreno hasta mesas de trabajo sectoriales y consultas vecinales. Por último, en Coyhaique se observó un alto nivel de implementación tanto de la ley de transparencia y acceso a la información como de la formalización de diversos mecanismos participativos tanto de información como de consulta e involucramiento, con lo cual se incluye como caso de control.

En cuanto a las similitudes para la selección, los tres casos son capitales de las regiones que conforman la Patagonia chilena, con lo cual se mantiene cierto nivel de homogeneidad en cuanto a los aspectos sociales y culturales. Respecto a su nivel de desarrollo y urbanización, aunque existen diferencias evidentes en cuanto a población e ingresos (Tabla 3), los tres casos son consideradas del tipo 2 por la Subsecretaría de Desarrollo Regional, siendo parte del grupo de 37 comunas mayores de desarrollo medio de Chile (SUBDERE 2016). 
Tabla 3: Datos socioeconómicos para 2016 de los casos seleccionados

\begin{tabular}{cccc} 
Municipalidad & Población & Ingresos totales & $\begin{array}{c}\text { Tipo } \\
\text { SUBDERE }\end{array}$ \\
\hline Coyhaique & 61.081 & $\$ 95.611 .145 .000$ & Tipo 2 \\
Punta Arenas & 130.738 & $\$ 235.742 .751 .000$ & Tipo 2 \\
Puerto Montt & 248.230 & $\$ 323.536 .247 .000$ & Tipo 2 \\
Fuente: Elaboración propia a partir de datos de SINIM & SUBDERE
\end{tabular}

Además, las tres ciudades eran gobernadas, durante la realización del trabajo de campo, por alcaldes pertenecientes a la coalición de la centroizquierda chilena (Nueva Mayoría): en Punta Arenas y Coyhaique eran alcaldes independientes con pacto con dicha coalición, y en Puerto Montt gobernaba el Partido Socialista.

Teniendo en cuenta los objetivos de investigación, se opta por un análisis cualitativo que permita observar a profundidad la implementación de dicha normatividad en las ciudades seleccionadas. Para ello, se realizan entrevistas semiestructuradas con actores clave tanto en la administración pública local como en las organizaciones de la sociedad civil, buscando cumplir con criterios intensivos de validez, dadas por la cercanía del entrevistado con las acciones que nos competen (Gaínza Veloso 2006). El trabajo en terreno se realizó entre abril de 2016 y febrero de 2017, y se realizaron 19 entrevistas en total en las tres ciudades seleccionadas (ver anexo). Una vez realizado el trabajo en terreno, se transcribieron y codificaron todas las entrevistas y, partiendo de nuestro marco teórico, y de los principios de la teoría fundamentada (Gaínza Veloso 2006, Jones, Manzelli y Pecheny 2007), se establecieron tres categorías para el análisis: efectos en la gestión de proximidad; efectos en la gestión transparente; y efectos en la gestión participativa. Para la sistematización se utilizó el programa de análisis cualitativo Atlas Ti. Se presentan a continuación los hallazgos encontrados.

\section{HALLAZGOS: EFECTOS EN LA GESTIÓN DE PROXIMIDAD}

Luego del análisis, se detectan tres efectos, evidenciables en los tres casos estudiados, en cuanto a la forma como las municipalidades se organizan internamente para la interacción con la ciudadanía: la desarticulación entre los mecanismos de transparencia y participación; la baja coordinación entre las diversas unidades de las municipalidades; y el gran protagonismo de los esfuerzos individuales de los funcionarios públicos. 
En efecto, en ninguno de los casos existe una relación visible entre las unidades de transparencia ni con los departamentos de organizaciones comunitarias, ni con los mecanismos de participación ciudadana. Es más, el trabajo de los funcionarios encargados de transparencia se centra, básicamente, en mantener las páginas web actualizadas (transparencia activa) y gestionar entre las diversas divisiones de la municipalidad todas las respuestas a las solicitudes de información (transparencia pasiva). No se evidencia, entonces, un esfuerzo sistemático por mantener un flujo constante de información entre la municipalidad y la ciudadanía a través de los mecanismos formales de transparencia ni de participación ciudadana, algo que se observa contrario a la idea misma de la gestión de proximidad.

Además, se observa un alto desaprovechamiento del papel articulador que podrían tener las unidades de transparencia: si es su misión mantener contacto constante con las diversas unidades de la municipalidad para poder resolver las solicitudes de información provenientes de la ciudadanía, perfectamente podría utilizarse dicha infraestructura institucional para gestionar y coordinar acciones al interior de la municipalidad, que permita evitar duplicidades y focalizar mejor los esfuerzos de la acción gubernamental. Esto, lamentablemente, no se presenta en ninguno de los casos estudiados. En cuanto a la participación ciudadana, se observa una desconexión evidente en los tres casos entre los departamentos de organizaciones comunitarias (pertenecientes a las Direcciones de Desarrollo Comunal DIDECO) y los COSOC (dependientes de la Secretaría Municipal), no solo en el organigrama, sino también en la práctica:

[...] el COSOC, más bien es visto por el Secretario Municipal, nosotros no hemos tenido mucha injerencia en ello a través del área de organizaciones comunitarias, que debió haber sido así. Pero, como la ley reconoce la figura del Secretario Municipal como articulador entre el municipio y del Consejo, la verdad es que no hemos sido muy convocados a ello (Entrevista 8, 22 de abril de 2016).

Por último, al preguntar tanto a funcionarios por su trabajo, así como a organizaciones sociales sobre su relación con la municipalidad, se evidenció que la motivación y la iniciativa de los funcionarios es fundamental para la gestión interna y externa de la municipalidad. En el caso de la transparencia, el buen funcionamiento de dichas unidades (que en todos los casos contaban con menos de tres funcionarios) depende de la relación que tenga el funcionario con las jefaturas de las distintas unidades municipales. El caso de Punta Arenas es muy claro en este punto: la encargada de dicho trabajo, quien al momento de la entrevista contaba con treinta años de experiencia en la municipalidad, conocía al detalle a los 
funcionarios y el funcionamiento de la administración local, lo cual, sumado al reconocimiento entre sus pares, le permitía gestionar las respuestas de manera rápida y oportuna. Gracias a su trabajo, la municipalidad se encuentra entre los mejores puntajes en materia de transparencia a nivel nacional.

En cuanto a la ley de participación, ha sido la iniciativa y el empeño de los funcionarios encargados de estos temas, y su constante interacción con la comunidad, los que han logrado crear y consolidar espacios de interacción efectiva entre la ciudadanía y la municipalidad. Tal es el caso de los Presupuestos Participativos Juveniles de Puerto Montt, así como la implementación de la Mesa de Planificación Urbana en Coyhaique.

\section{HALLAZGOS: EFECTOS EN LA GESTIÓN TRANSPARENTE}

En cuanto a los mecanismos de transparencia activa y acceso a la información, durante el trabajo en terreno se observaron tanto avances como desafíos.

Por un lado, se evidencia una visión positiva de ciertos aspectos de la ley de transparencia y acceso a la información, como lo son la creación de páginas web con información municipal y las solicitudes de información pública. Tanto los funcionarios como los miembros de la sociedad civil dan cuenta del efecto positivo que ha significado la existencia de dichos mecanismos, los cuales son detallados como herramientas para acercar a los ciudadanos a su administración local.

Por el otro, también se observan críticas sobre la calidad y la profundidad de la información otorgada a través de dichos mecanismos, observándose también un nivel alto de desconfianza en la municipalidad. Incluso en el caso de Punta Arenas, siendo una administración que ha estado históricamente entre los mejores del país en cuanto a transparencia activa y acceso a la información, varios miembros de organizaciones sociales mantenían una visión negativa de su municipalidad y un alto nivel de desconfianza con los datos de transparencia, sea porque la información que les ha sido entregada les resulta insuficiente, o porque los procesos de solicitud de información les resultan lentos y engorrosos, o porque se espera un nivel más minucioso de información de proyectos específicos.

Ahora, el mayor problema que se evidenció en la investigación es el alto desconocimiento y el bajo uso, por parte de las organizaciones sociales, de este tipo de herramientas para el acceso a la información. Aunque los entrevistados de la sociedad civil, por lo general, conocen la página web 
de la municipalidad y la opción de solicitar información, son pocos los que manifiestan haber usado dichos mecanismos. En las entrevistas a funcionarios se mencionó, en varias ocasiones, cómo dichos mecanismos son utilizados más por académicos, o por empresas privadas (sobre todo de construcción) que por organizaciones de la sociedad civil:

Básicamente yo creo que esto dio apertura a mucha gente que hace investigaciones, estudios, y expresamente lo ponen en la mayoría de los casos, entonces yo creo que por ahí se desvirtúa un poco la [sic] como para que uno pueda tener claridad en el perfil que es realmente la comunidad el ciudadano común y corriente que está interesado en tener claridad sobre algunos temas, yo diría que no va por ese lado [...] cuando piden información del sistema de información geográfica, entonces lo piden empresas privadas. Entonces decía, pero porque nosotros tenemos que entregarle algo si después estos otros van a lucrar con esto, es un trabajo nuestro que estamos pagando (Entrevista 1, 16 de abril 2016).

[...] lo que más preguntan es por temas de terrenos, planos, ese tipo de cosas, habitualmente llegan de esos aquí, por ejemplo, solicitud de presupuesto municipal en materias de seguridad, solicita copia de plano, lo que más solicitan son planos (Entrevista 12, 25 abril 2016). Preguntan de todo, desde el listado de las patentes comerciales, patentes de alcoholes, eh no sé... viajes del Alcalde, viáticos, remuneraciones, actas de consejo, audios de consejo, eh ¿qué más? No sé, de distintas cosas. Desde las cosas más insólitas, una vez preguntaron sobre estudios de los hongos silvestres que había en Coyhaique. No sé, de muchas cosas preguntan (Entrevista 17, 23 de febrero 2017).

A su vez, al entrevistar a líderes locales, se evidenciaron algunos aspectos que podrían afectar el uso de estos mecanismos, que van desde aspectos de brecha digital (sobre todo en localidades rurales) hasta la preferencia de las organizaciones por hacer conversaciones personales e ir directamente a la municipalidad para obtener información.

Por último, al solicitarles algunas ideas para mejorar su trabajo, los funcionarios encargados de transparencia entrevistados coinciden en una recomendación: la necesidad de mejorar los sistemas de archivo y gestión documental, de manera que se pueda mejorar la gestión interna de información, no solo de cara a la ciudadanía, sino para el mismo trabajo interno de la municipalidad. 


\section{HALLAZGOS: EFECTOS EN LA GESTIÓN PARTICIPATIVA}

En cuanto a la ley de participación ciudadana, cada uno de los casos estudiados había implementado, de manera diferenciada, sus propias estrategias de participación e interacción con las organizaciones sociales territoriales (juntas de vecinos) y funcionales (organizaciones culturales y temáticas). Un elemento diferenciador entre los casos de estudio, muy presente en la investigación, es la figura de los Consejos Comunales de Organizaciones Sociales (COSOC).

Creados por la Ley 20.500, los COSOC son mecanismos colegiados de participación ciudadana en la gestión pública, cuyos miembros son representantes elegidos por votación entre las organizaciones sociales registradas en la municipalidad, y que tiene como objetivo permitir que la sociedad civil tenga incidencia en el diseño, la implementación y la evaluación de políticas públicas a nivel local. Aunque existe un énfasis legal en la importancia de estos mecanismos, los COSOC son criticados, en los tres casos de estudio, por dos características fundamentales: su carácter consultivo, que impide que sus decisiones sean vinculantes para la administración local, y el hecho de ser presididas por el Alcalde, lo que genera una alta dependencia en la discrecionalidad de dicha autoridad.

El caso de Puerto Montt es muy indicativo de esto: aun siendo la ciudad intermedia que cuenta con el mayor número de mecanismos en su ordenanza de participación, al momento de realizar la investigación en terreno, no se había constituido el COSOC, y en las entrevistas se evidenció que existía un desinterés manifiesto, tanto de las organizaciones sociales como de los funcionarios municipales, por conformar dicho mecanismo, ya que lo consideraban engorroso, burocrático y sin mayores beneficios para las partes. En cambio, al interior de la municipalidad se evidencia un modelo de gestión para atender a las organizaciones sociales de manera directa, previo al surgimiento de la Ley 20.500, a través de oficinas sectoriales y poblacionales de su Dirección de Desarrollo Comunal (DIDECO) y del Departamento de Organizaciones Comunitarias de la misma dirección. Dicha interacción directa es vista de manera muy positiva tanto por los funcionarios como por los líderes locales entrevistados, aunque dicho esquema podría, según mencionan entrevistados de la municipalidad y de organizaciones sociales, dejar la puerta abierta para el uso político y el clientelismo, dado que permite un alto nivel de discrecionalidad en las autoridades que designan apoyos a las organizaciones sociales.

En cambio, la formalidad de la legislación se ha cumplido a cabalidad tanto en Punta Arenas como en Coyhaique. Aun así, se observa una 
diferencia esencial entre estos dos casos: la percepción del funcionamiento de dicha institucionalidad. Mientras en Coyhaique se muestra como un gran avance para la interacción entre la municipalidad y las organizaciones sociales, a pesar de algunas complejidades para la movilización ciudadana (desconocimiento de los mecanismos, falta de interés de la ciudadanía), en Punta Arenas se observó una visión positiva de los consejeros más nuevos, pero una gran frustración entre los que llevaban más tiempo siendo parte del Consejo. Dicho desgaste se presenta, según los consejeros entrevistados, dadas las altas expectativas que se crearon alrededor de la nueva institucionalidad, donde esperaban encontrar una participación más activa y vinculante, para luego encontrarse con mecanismos consultivos y en cierta medida instrumentalizados:

Antes de entrar al COSOC estuvieron varios colegas dirigentes allí en esa [sic], estuvieron varios, y todos salieron desencantados y por lo mismo nadie quiso presentarse en estas elecciones y con decirte que hubo, tan desencantados estaban que al presentarme yo fue algo fortuito porque yo pasé a hacer un trámite a la municipalidad y se me había olvidado que había elecciones, entonces empecé a llamar rápido a los dirigentes y me dicen no, yo no quiero saber nada de eso porque no sirve (Entrevista 7, 22 de abril, 2016).

A parte de los problemas de diseño institucional mencionados, también se evidenció una serie de desafíos en cuanto a la participación ciudadana a nivel local. En primer lugar, la baja participación y en algunos casos el claro desconocimiento de los ciudadanos frente a los mecanismos de participación. In en los casos con alto nivel de cumplimiento formal, la observación con más menciones de parte de los entrevistados (tanto funcionarios como dirigentes) es el bajo interés que genera la participación entre la ciudadanía:

No creo que nos pase solamente a nosotros. Yo creo que la crisis de participación es un tema a nivel nacional y quizás me atrevería a decir que traspasa nuestras fronteras, pero básicamente en nuestra comuna la participación de un sector de rango etario que va desde los 18 a los 40 es muy muy baja, es muy muy baja (Entrevista 3, 19 abril 2016).

Algunas razones esgrimidas en las entrevistas dan cuenta de varias posibles causas: la rigidez de los mecanismos de participación, la desconfianza que generan las instituciones estatales y la política en general, el costo que genera la participación presencial, la falta de incidencia de los mecanismos en la vida pública, los pocos recursos con los que cuentan dichas instancias, y el bajo reconocimiento que tienen los mecanismos de 
participación entre las organizaciones sociales y la ciudadanía en general. Todas estas situaciones, que en parte se producen por la misma legislación, desincentivarían la participación ciudadana y generarían un nuevo ciclo de desconfianza en las instituciones.

Un segundo desafío, que se observa en los tres casos, es el papel de los dirigentes sociales, específicamente de los presidentes de las juntas de vecinos. Tanto los funcionarios como los mismos dirigentes entrevistados mencionan dos situaciones críticas: por un lado, la baja capacidad de movilización de los dirigentes con sus bases, en ocasiones porque no logran generar espacios de vinculación con nuevas generaciones, en otras por un claro desinterés de los dirigentes por hacerlo; y por el otro, se evidencian casos de dirigentes que instrumentalizan sus funciones por intereses políticos, estableciendo redes clientelares en sus territorios. Aunque también se evidenciaron comentarios a favor del trabajo realizado por los dirigentes en sus territorios, sobre todo en Puerto Montt y en Coyhaique, la figura de las juntas de vecinos resulta conflictiva y problemática en los tres casos estudiados.

Ahora, cabe resaltar que en los tres casos de estudio han surgido estrategias propias de participación ciudadana que, para los entrevistados, se muestran más útiles y eficientes que el mismo COSOC: en el caso de Coyhaique se estableció el Directorio Urbano, una iniciativa con participación de organizaciones sociales, la academia y los diversos estamentos del gobierno central en la región, que busca la interacción entre diversos sectores para la elaboración del Plan Centenario, una planificación estratégica unificada de cara a la realización de obras de infraestructura de cara a los cien años de la ciudad, en 2029; en Punta Arenas, la Dirección de Organizaciones Comunitarias de la DIDECO ha realizado de manera continua una estrategia de presupuestos participativos con enfoque territorial, un mecanismo que ha permitido atender de manera diferenciada los diversos sectores de la ciudad. En este último caso, la ventaja de esta estrategia es su capacidad de adaptación al entorno, sin la rigidez que implica la institucionalización de la participación vía ordenanza municipal, con lo cual las acciones pueden focalizarse a sectores de la población que no necesariamente tienen interés en formalizarse. Algo similar ocurre en Puerto Montt, donde se ha implementado desde el ańo 2011 el programa de Presupuestos Participativos Juveniles, un programa que busca apoyar iniciativas propias de organizaciones sociales juveniles, un segmento poblacional que no suele encontrarse representado en las estructuras tradicionales de la participación institucional. 
Tabla 4: Hallazgos de investigación

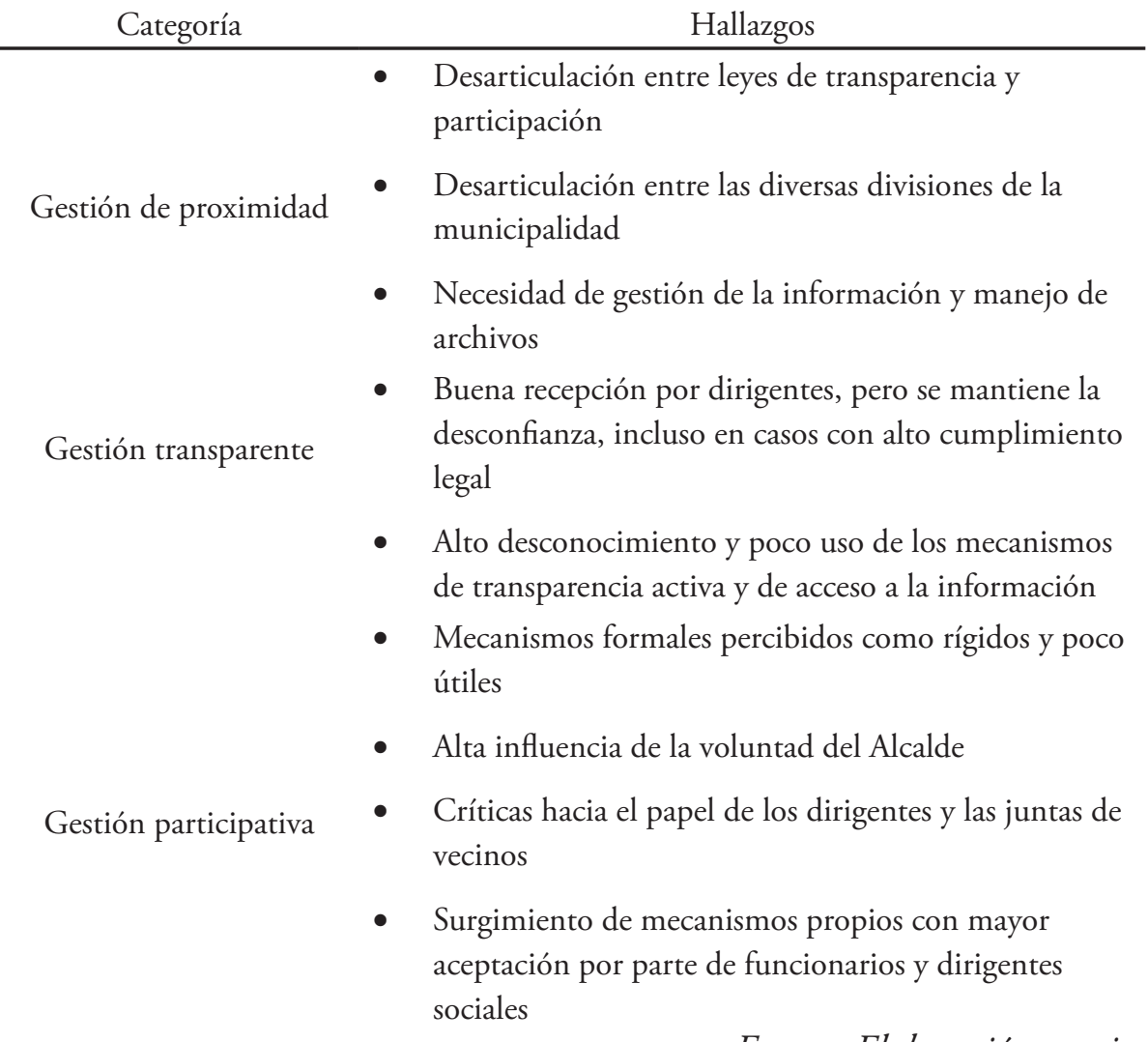

Fuente: Elaboración propia.

\section{DISCUSIÓN}

Luego de la revisión de las entrevistas y el detalle de los hallazgos, y mirando a la luz de las premisas teóricas de la gobernanza democrática local (proximidad, transparencia y gestión participativa) descritos en el marco teórico, se evidencia que, a pesar de los avances que se observan tanto en la institucionalidad formal como en la implementación de las leyes de transparencia, acceso a la información y participación ciudadana, la situación de la gobernanza local en Chile aún se encuentra lejos del ideal. En cuanto a la premisa de proximidad, aun cuando el surgimiento de mecanismos institucionales de transparencia y participación es visto como un gran avance -que redunda en una mayor capacidad de la ciudadanía para acceder a la información pública, así como nuevos escenarios de retroalimentación para la administración local- se observa a su vez una serie de problemas en la implementación, los que evitan el aprovechamiento de dichos mecanismos en la escala municipal. En primer 
lugar, la desarticulación interna, tanto de los mecanismos de participación y los de transparencia, como entre las diversas divisiones municipales, se convierten en puntos de bloqueo no solo para la gobernanza democrática, sino también para la gestión pública local. Implicaría entonces que, según los casos estudiados, la gestión interna de la municipalidad es un factor importante para el desarrollo de estrategias de gobernanza a nivel local, algo que ya ha sido estudiado en otras latitudes (Smith 2009). Para el caso de Chile esto es importante, puesto que en 2016 se expide la Ley 20.922 sobre fortalecimiento de la gestión y profesionalización del personal municipal, la cual permite a las municipalidades crear y modificar sus plantas municipales según sus propias necesidades. Con esto, las administraciones locales tendrían la capacidad de diseñar estrategias de articulación y coordinación interna que fomenten la gobernanza democrática local.

Otro punto a tener en cuenta es el diseño institucional de dichos mecanismos. En el caso chileno, tanto los espacios institucionales de transparencia como los de participación son externos al nivel municipal, viniendo sus lineamientos marcados desde la legislación nacional con no pocas rigurosidades, algo que evita su adaptación a los diversos entornos locales. Dicha rigidez normativa, a la larga, termina traduciéndose en el cumplimiento formal de las disposiciones legales, pero no necesariamente en el desarrollo de mecanismos de gobernanza local. Al final, la implementación de los espacios (sobre todo los de participación ciudadana) dependen directamente de la voluntad política del Alcalde, un punto señalado como determinante en el detallado estudio de Merilee Grindle (2007) sobre las causas de éxito y fracaso de la gobernanza local. Todo esto puede terminar generando una gran frustración entre los actores participantes, quienes se acercan a estos espacios con la promesa de un mayor acceso tanto a la información como a la toma de decisiones, para encontrarse enfrentados con mecanismos cerrados y basados en la voluntad de las autoridades locales. En otras palabras, la forma puede que se cumpla, pero la esencia de la gobernanza democrática, donde la interacción busca una vinculación sustantiva tanto a la información como al proceso mismo de toma de decisiones, queda aún sin desarrollarse.

En cuanto a la premisa de transparencia y apertura, los hallazgos marcan varios avances dentro de la administración pública, aunque aún hay mucho que enfrentar una cultura del "secretismo" y la desarticulación de las diversas divisiones de las municipalidades estudiadas. En efecto, durante la investigación se evidenció que no se ha otorgado el nivel de relevancia que se merece al trabajo de las unidades de transparencia, las cuales podrían actuar como articuladoras de la información pública al interior de las municipalidades. Existen múltiples oportunidades para aprovechar este 
trabajo, tanto para el mejoramiento de la gestión documental, como para robustecer la gestión interna de las municipalidades.

Otro punto por mencionar, que implica también a las organizaciones de la sociedad civil, es la ausencia, en los tres casos estudiados, de las llamadas comunidades interpretativas, entendidas como grupos de ciudadanos especializados en el manejo de la información pública para alimentar el debate y la deliberación social. Esto podría explicarse en parte por el alto desconocimiento (o desinterés) de parte de las organizaciones sociales en particular, y la ciudadanía en general, de los mecanismos de transparencia activa y acceso a la información, así como a la desarticulación de ambas políticas en lo loca. Aunque en la teoría, la transparencia y el acceso a la información deben ser esenciales para una participación ciudadana informada, en la práctica no se evidencia una articulación entre ambas políticas, sea por desarticulación al interior de las municipalidades, o sea por la falta de iniciativa de las organizaciones sociales. Esto recuerda los comentarios de Fox (2007) sobre la relación incierta entre la transparencia y la rendición de cuentas, mencionando que no necesariamente la existencia de mecanismos de transparencia genera espacios participativos o movilización ciudadana para la responsabilidad pública. El trabajo de Worthy (2010) sobre la no tan clara relación entre transparencia y confianza también arroja luces sobre esta situación: la existencia de una legislación que regule la información pública no logra, por sí sola, generar confianza ni movilizar a la población hacia mecanismos participativos. Se requiere entonces de una mayor movilización de la sociedad civil para aprovechar las potenciales ventajas de la apertura de información pública a nivel municipal.

En cuanto al principio de una gestión participativa, se observan varios desafíos a enfrentar, aún en los casos con mayor cumplimiento. La baja participación ciudadana, el papel en ocasiones monopólico del capital político por parte de dirigentes sociales territoriales, la desarticulación que se presenta con las bases sociales y la percepción de inoperancia de los mecanismos institucionales de participación son problemas evidentes en todos los casos. Retomando el punto sobre la proximidad administrativa, la rigidez de los mecanismos institucionales creados por la ley ha sido fuente de frustración entre los pocos que sí participan, lo cual genera una sensación de inoperancia de la participación, un efecto contraproducente que las legislaciones sobre participación generan no solo en Chile, sino que se observan en otros países como España (Ruano 2010) o Colombia (Hernández-Bonivento 2017). 
Ahora, no todo son malas noticias: ante las rigideces legales, las tres municipalidades estudiadas han logrado desarrollar estrategias propias de participación ciudadana, las cuales son descritas por los entrevistados como herramientas mucho más útiles y efectivas que los mecanismos tradicionales. Dicha percepción sobre los mecanismos propios, como los presupuestos participativos territoriales en Punta Arenas, los PPJ en Puerto Montt, o como el Directorio Urbano de Coyhaique, demuestra la importancia de fomentar e incentivar este tipo de estrategias, las cuales podrían enfocarse hacia las propias necesidades locales, mejorando su eficacia y generando una sensación de utilidad entre los usuarios, además de establecer cierta apropiación de los mecanismos deliberativos por parte de los actores sociales involucrados. Aunque es importante que dichas estrategias locales se implementen con rigurosidad en las formas, y que establezcan claramente sus propias reglas del juego (en aras de evitar instrumentalizaciones y discrecionalidades), estos ejercicios se muestran más adecuados para la implementación de estrategias de gobernanza democrática, al evitar caer en rigideces inhabilitantes y fomentar, a través de la apropiación de dichos espacios por parte de los funcionarios y la ciudadanía en general, el diálogo, el debate y la deliberación a nivel local.

No podríamos cerrar el presente trabajo de investigación sin mencionar líneas de trabajo a futuro, como sería la necesidad de mejorar los mecanismos de evaluación y seguimiento de estas leyes, para lograr hacer una visión integral de la gobernanza a nivel local. Quedarnos en la formalidad de la implementación implica perder las múltiples oportunidades que presentan la transparencia, el acceso a la información y la participación ciudadana en las esferas locales de gobierno. También es necesario señalar las limitaciones de la presente investigación, centrada en un tipo específico de municipalidad (las ciudades intermedias), por lo que sería interesante también analizar el comportamiento de estos mecanismos en comunas altamente urbanizadas frente a comunas rurales. Por último, sería fundamental observar el funcionamiento de los mecanismos propios de participación ciudadana, de manera que podamos no solo identificar buenas prácticas, sino entender mejor sus alcances y potencialidades en la escala local de gobierno. 


\section{REFERENCIAS}

Aguilar Villanueva, L. (2008). Gobernanza y Gestión Pública. México: Fondo de Cultura Económica.

Blanco, I. y Gomá, R. (2002). Proximidad y Participación: Marco Conceptual y Presentación de Experiencias. En Blanco, I. y Gomá, R. (Eds.), Gobiernos Locales y Redes Participativas. Barcelona: Ariel Social.

Brugué, J. y Gallego, R. (2007). ¿Una Administración Pública Democrática? En Font, J. (Ed.), Ciudadanos y Decisiones Públicas. Barcelona: Ariel Ciencia Política.

Calvo, E. y Murillo, M. V. (2013). When Parties Meet Voters: Assessing Political Linkages Through Partisan Networks and Distributive Expectations in Argentina and Chile. Comparative Political Studies 46 (7), 851-882.

Carmona, R. R. (2005). Nuevas Formas de Gobierno y Gestión Pública en el Escenario Local: Elementos y Perspectivas para el Estímulo de Procesos Concertados de Desarrollo. Buenos Aires: Asociación de Administradores Gubernamentales.

Consejo Nacional de Participación Ciudadana y Fortalecimiento de la Sociedad Civil (2017). Estado de la participación ciudadana en Chile y propuestas de reforma a la Ley 20.500 sobre Asociaciones y Participación Ciudadana en la Gestión Pública. Disponible en: www. consejoparticipacion.cl/content/uploads/2017/01/informe-final.pdf [31-12-2018].

Consejo para la Transparencia (2017). Informe de Transparencia Municipal. Santiago: CPLT. Disponible en: http://itm.cplt.cl/ [31-12-2018].

Cornford, J., Wilson, R., Baines, S. y Richardson, R. (2013). Local Governance in the New Information Ecology: The Challenge of Building Interpretative Communities. Public Money \& Management, 33 (3), 201-208.

De Sousa Santos, B. (2003). Democracia y Participación: El Ejemplo del Presupuesto Participativo de Porto Alegre. Barcelona: El Viejo Topo.

Delamaza, G., Robles, C., Montecinos, E. y Ochsenius, C. (2012). Redes de Política Pública y Agendas de Participación Ciudadana en el Chile 
Postransicional ¿Desafiando la Política o Recreando sus Límites? Gestión y Politica Pública, XXI (1), 45-86.

Escoffier, S. (2017). Policy Metaphors and Deep Local Democracy: The Case of the Chilean Neighborhood Recovery Program. Revista Iberoamericana de Estudios Municipales, 15, 35-64.

Evans, A. y Campos, A. (2012). Open Government Initiatives: Challenges of Citizen Participation. Journal of Policy Analysis and Management, 32 (1), 172-185.

Falleti, T. G. (2010). Decentralization and Subnational Politics in Latin America. Cambridge: Cambridge University Press.

Font, J. (2007). Ciudadanos y Decisiones Públicas. Barcelona: Ariel Ciencia Política.

Fox, J. (2007). The Uncertain Relationship Between Transparency and Accountability. Development in Practice, 17 (4-5), 663-671.

Fung, A. (2006). Varieties of Participation in Complex Governance. Public Administration Review 66 (special issue), 66-75.

. (2015). Putting the Public Back into Governance: The Challenges of Citizen Participation and Its Future. Public Administration Review, 75 (4), 513-522.

Gaínza-Veloso, A. (2006). La Entrevista a Profundidad Individual. En Canales, C. (Ed.), Metodologías de Investigación Social. Santiago: LOM.

Geurtz, C. y van de Wijdeven, T. (2010) Making Citizen Participation Work: The Challenging Search for New Forms of Local Democracy in the Netherlands. Local Government Studies, 36 (4), 531-549.

Goss, S. (2001). Making Local Governance Work: Networks, Relationships and the Management of Change. Nueva York: Palgrave.

Grindle, M. S. (2007). Going Local. Decentralization, Democratization and the Promise of Good Governance. Princeton: Princeton University Press.

Harrison, T. M. y Sayogo, D. S. (2014). Transparency, Participation, and Accountability Practices in Open Government: A Comparative Study. Government Information Quarterly, 31 (4), 513-525. 
Hernández-Bonivento, J. (2017). Análisis de Mecanismos de participación Local en Colombia: Audiencias de Rendición de Cuentas y Consejos Territoriales de Planeación. Civilizar Ciencias Sociales y Humanas, 17 (32) 67-80.

Iacoviello, M. y Strazza, L. (2014) Diagnóstico del Servicio Civil en América Latina. En: Cortázar, J. C., Lafuente, M. y Sanginés, M. (Ed.), Al Servicio del Ciudadano. Una Década de Reformas del Servicio Civil en América Latina (2004-13). Washington: Banco Interamericano de Desarrollo.

Jones, D., Manzelli, H. y Pecheny, M. (2007). La Teoría Fundamentada: Su Aplicación en una Investigación sobre Vida Cotidiana con VIH/Sida y con Hepatitis C. En Kornblit, A. L. (Ed.), Metodologías Cualitativas en Ciencias Sociales. Modelos y Procedimientos de Análisis. Buenos Aires: Editorial Biblos.

Kooiman, J. (2003). Governing as Governance. London: SAGE.

Lathrop, D. y Ruma, L. (2010). Open Government: Collaboration, Transparency and Participation in Practice. Sebastopol: O'Reilly Editors.

Levi-Faur, D. (2012). From "Big Government" to "Big Governance"? En Levi-Faur, D. (Ed.), The Oxford Handbook of Governance. Oxford: Oxford University Press.

Lourenco, R. (2015). An Analysis of Open Government Portals: A Perspective of Transparency for Accountability. Government Information Quarterly, 32 (3), 323-332.

Mascareño, C. (2008). Descentralización y Democracia en América Latina: ¿Una Relación Directa? Elementos Conceptuales para su Estudio. College Park: Latin American Studies Center, University of Maryland, College Park.

Maturana, F. y Rojas, A. (2015). Ciudades Intermedias: Territorios Olvidados. Santiago: RIL Editores.

Mcnulty, S. (2013). Participatory Democracy? Exploring Peru's Efforts to Engage Civil Society in Local Governance. Latin American Politics and Society, 55 (3), 69-92. 
Meijer, A., Curtin, D., y Hillebrandt, M. (2012). Open Government: connecting vision and voice. En International Review of Administrative Sciences, 78, 10-29.

Michels, A. y De Graaf, L. (2010). Examining Citizen Participation: Local Participatory Policy Making and Democracy. Local Government Studies, 36 (4), 477-491.

Navarro Gómez, C. (2002). Gobernanza en el ámbito local. VII Congreso Internacional del CLAD sobre Reforma del Estado y de la Administración Pública. Lisboa: CLAD.

Navarro, C. (2004). Sociedades Politicas Locales: Democracia Local y Gobernanza Multinivel. Madrid: Universidad Autónoma de Madrid.

Navarro, C. J. y Ramírez, M. A. (2005). Una Propuesta para el Análisis de la Acción de los Gobiernos Locales. Revista de Estudios Politicos, 128, 161-177.

Olavarría, M. (2010). ¿Cómo se Formulan las Politicas Públicas en Chile? Tomo I. Santiago: Editorial Universitaria.

Osborne, S. P. (2010). The New Public Governance? Emerging Perspectives on the Theory and Practice of Public Governance. Nueva York: Routledge.

Paredes, J. P. (2007). Otra Democracia: Sociedad Civil, Ciudadanía y Gobernanza Local. Notas para la Discusión. Polis, 5 (16), 445-474.

Peters, B. G. (2010). Meta-Governance and Public Management. En Osborne, S. P. (Ed.), The New Public Governance? Emerging Perspectives on the Theory and Practice of Public Governance. Nueva York: Routledge.

Prats, J. (2005). De la Burocracia al Management, del Management a la Gobernanza. Madrid: INAP.

Ruano, J. M. (2010). Contra la Participación: Discurso y Realidad de las Experiencias de Participación Ciudadana. Política y Sociedad, 47 (3), 93-108.

Salvador Crespo, M. (2008). El Fortalecimiento Institucional de los Municipios. En Castillo Blanco, F. (Ed.), Análisis de los Procesos de Descentralización en América Latina: Balances y Perspectivas. Granada: Unión Iberoamericana de Municipalistas. 
Sistema Nacional de Información Municipal (SINIM) (2018). Datos Municipales. Disponible en: http://datos.sinim.gov.cl/datos municipales.php [31-12-2018].

Smith, C. R. (2009). Institutional Determinants of Collaboration: An Empirical Study of County Open-Space Protection. Journal of Public Administration Research and Theory, 19 (1), 1-21.

Subsecretaría de Desarrollo Regional y Administrativo (SUBDERE) (2016). Resolución 82 de 2016. Disponible en: http://www.sinim.gov.cl/ desarrollo local/figem/archivos/Resolucion\%2082\%20FIGEM\%20 2016 TTT.pdf [31-12-2018].

Subirats, J. (2007). Nuevos Mecanismos de Participación y Democracia: Promesas y Amenazas. En Font, J. (Ed.), Ciudadanos y Decisiones Públicas. Barcelona: Ariel Ciencia Política.

Torfing, J. (2012). Governance Networks. En Levi-Faur, D. (Ed.), The Oxford Handbook of Governance. Oxford: Oxford University Press.

United Nations Development Program (UNPD). (2010). Building Bridges Between the State and the People: An Overview of Trends and Developments in Public Administration and Local Governance. Washington: UNPD.

Varela Álvarez, E. J. (2010). Gestión y Gobernanza Local en Perspectiva Comparada: Las Politicas Públicas de Modernización Administrativa en los Gobiernos Locales de Galicia y el Norte de Portugal. Tesis (Doctorado en Ciencia Política), Universidad Complutense de Madrid, Madrid, España.

Vial, M. C. (2016). Las Municipalidades en Chile. En Ruano J. M. y Vial, M. C. (Eds.), Manual de Gobiernos Locales en Iberoamérica. Santiago: CLAD, Universidad Autónoma de Chile.

Villoria, M. (2010). La Democratización de la Administración Pública: Marco Teórico. En Ruiz-Huerta J. y Villoria, M. (Eds.), Gobernanza Democrática y Fiscalidad. Madrid: Tecnos.

Worthy, B. (2010). More Open but Not More Trusted? The Effect of the Freedom of Information Act 2000 on the United Kingdom Central Government. Governance. An international Journal of Policy, Administration, and Institutions, 23 (4), 561-582. 
Yavuz, N. y Welch, E. W. (2014). Factors Affecting Openness of Local Government Websites: Examining the Differences Across Planning, Finance and Police Departments. Government Information Quarterly, 31 (4), 574-583.

Yilmaz, S., Beris, Y. y Serrano-Berthet, R. (2008). Local Government Discretion and Accountability: A Diagnostic Framework for Local Governance. Washington: Social Development Working Papers, World Bank.

Young, I. M. (2002) Inclusion and Democracy. Oxford: Oxford University Press.

Recibido: 15-11-2018

Aceptación de la versión final: 31-12-2018 


\section{ANEXO}

\section{Listado de entrevistas}

\begin{tabular}{|c|c|c|c|}
\hline Número & Perfil & Lugar & Fecha \\
\hline 1 & $\begin{array}{c}\text { Funcionario de } \\
\text { Transparencia municipal }\end{array}$ & $\begin{array}{c}\text { Punta Arenas, Región } \\
\text { Magallanes }\end{array}$ & $\begin{array}{l}18 \text { de abril de } \\
2016\end{array}$ \\
\hline 2 & Consejero COSOC & $\begin{array}{c}\text { Punta Arenas, Región } \\
\text { Magallanes }\end{array}$ & $\begin{array}{l}19 \text { de abril de } \\
2016\end{array}$ \\
\hline 3 & $\begin{array}{l}\text { Funcionario de } \\
\text { Organizaciones } \\
\text { Comunitarias }\end{array}$ & $\begin{array}{c}\text { Punta Arenas, Región } \\
\text { Magallanes }\end{array}$ & $\begin{array}{l}19 \text { de abril de } \\
2016\end{array}$ \\
\hline 4 & Consejero del COSOC & $\begin{array}{c}\text { Punta Arenas, Región } \\
\text { Magallanes }\end{array}$ & $\begin{array}{l}21 \text { de abril de } \\
2016\end{array}$ \\
\hline 5 & Consejero del COSOC & $\begin{array}{c}\text { Punta Arenas, Región } \\
\text { Magallanes }\end{array}$ & $\begin{array}{l}21 \text { de abril de } \\
2016\end{array}$ \\
\hline 6 & Consejero del COSOC & $\begin{array}{c}\text { Punta Arenas, Región } \\
\text { Magallanes }\end{array}$ & $\begin{array}{l}21 \text { de abril de } \\
2016\end{array}$ \\
\hline 7 & $\begin{array}{c}\text { Presidente de la Unión de } \\
\text { Juntas de Vecinos }\end{array}$ & $\begin{array}{c}\text { Punta Arenas, Región } \\
\text { Magallanes }\end{array}$ & $\begin{array}{l}22 \text { de abril de } \\
2016\end{array}$ \\
\hline 8 & Director de DIDECO & $\begin{array}{c}\text { Punta Arenas, Región } \\
\text { Magallanes }\end{array}$ & $\begin{array}{l}22 \text { de abril de } \\
2016\end{array}$ \\
\hline 9 & $\begin{array}{c}\text { Director de Organizaciones } \\
\text { Comunitarias }\end{array}$ & $\begin{array}{c}\text { Puerto Montt, Región } \\
\text { Los Lagos }\end{array}$ & $\begin{array}{l}25 \text { de abril de } \\
2016\end{array}$ \\
\hline 10 & $\begin{array}{c}\text { Funcionarios de Atención a } \\
\text { Jóvenes }\end{array}$ & $\begin{array}{c}\text { Puerto Montt, Región } \\
\text { Los Lagos }\end{array}$ & $\begin{array}{l}25 \text { de abril de } \\
2016\end{array}$ \\
\hline 11 & $\begin{array}{l}\text { Presidente de la Unión de } \\
\text { Juntas de Vecinos }\end{array}$ & $\begin{array}{c}\text { Puerto Montt, Región } \\
\text { Los Lagos }\end{array}$ & $\begin{array}{l}25 \text { de abril de } \\
2016\end{array}$ \\
\hline 12 & $\begin{array}{l}\text { Funcionario de } \\
\text { Organizaciones } \\
\text { Comunitarias }\end{array}$ & $\begin{array}{c}\text { Puerto Montt, Región } \\
\text { Los Lagos }\end{array}$ & $\begin{array}{l}25 \text { de abril de } \\
2016\end{array}$ \\
\hline 13 & $\begin{array}{l}\text { Miembro de una } \\
\text { Organización Funcional de } \\
\text { Jóvenes }\end{array}$ & $\begin{array}{c}\text { Puerto Montt, Región } \\
\text { Los Lagos }\end{array}$ & $\begin{array}{l}26 \text { de abril de } \\
2016\end{array}$ \\
\hline 14 & $\begin{array}{l}\text { Presidente de la Unión de } \\
\text { Juntas de Vecinos }\end{array}$ & $\begin{array}{c}\text { Puerto Montt, Región } \\
\text { Los Lagos }\end{array}$ & $\begin{array}{l}27 \text { de abril de } \\
2016\end{array}$ \\
\hline 15 & $\begin{array}{c}\text { Funcionario de } \\
\text { Transparencia municipal }\end{array}$ & $\begin{array}{l}\text { Puerto Montt, Región } \\
\text { Los Lagos }\end{array}$ & $\begin{array}{l}27 \text { de abril de } \\
2016\end{array}$ \\
\hline 16 & Secretario Municipal & $\begin{array}{c}\text { Coyhaique, Región de } \\
\text { Aysén }\end{array}$ & $\begin{array}{l}23 \text { de febrero } \\
\text { de } 2017\end{array}$ \\
\hline 17 & $\begin{array}{c}\text { Funcionario de } \\
\text { Transparencia municipal }\end{array}$ & $\begin{array}{l}\text { Coyhaique, Región de } \\
\text { Aysén }\end{array}$ & $\begin{array}{l}23 \text { de febrero } \\
\text { de } 2017\end{array}$ \\
\hline 18 & Consejera del COSOC & $\begin{array}{l}\text { Coyhaique, Región de } \\
\text { Aysén }\end{array}$ & $\begin{array}{l}23 \text { de febrero } \\
\text { de } 2017\end{array}$ \\
\hline 19 & Consejera del COSOC & $\begin{array}{c}\text { Coyhaique, Región de } \\
\text { Aysén }\end{array}$ & $\begin{array}{l}23 \text { de febrero } \\
\text { de } 2017\end{array}$ \\
\hline
\end{tabular}

